\title{
Editorial
}

\section{New Challenges in Fractional Systems}

\author{
Jocelyn Sabatier, ${ }^{1}$ Clara Ionescu, ${ }^{2}$ József K. Tar, ${ }^{3}$ and José A. Tenreiro Machado ${ }^{4}$ \\ ${ }^{1}$ IMS Laboratory, Bordeaux 1 University, 351 Cours de la Liberation, 33400 Talence, France \\ ${ }^{2}$ Ghent University, Technologiepark 913, B9052 Zwijnaarde, Belgium \\ ${ }^{3}$ John von Neumann Faculty of Informatics, Óbuda University, H-1034 Budapest, Hungary \\ ${ }^{4}$ Department of Electrical Engineering, Institute of Engineering, Polytechnic of Porto, 4200-072 Porto, Portugal
}

Correspondence should be addressed to Jocelyn Sabatier; jocelyn.sabatier@u-bordeauxl.fr

Received 9 May 2013; Accepted 9 May 2013

Copyright (C) 2013 Jocelyn Sabatier et al. This is an open access article distributed under the Creative Commons Attribution License, which permits unrestricted use, distribution, and reproduction in any medium, provided the original work is properly cited.

Without any doubt, the recently emerging tools from fractional calculus became successful in a manifold of applications and currently the playground of modern engineering sciences. Fractional order differentiation consists in the generalisation of classical integer differentiation to real or complex orders. From a mathematical point of view, several interpretations of fractional differentiation were proposed, but there is still a deep debate about it. However, all these interpretations demonstrate that fractional order differentiation cannot simply be connected to the slope at one point of the derived function for instance. This lack of interpretation is in fact due to the definition of the fractional order operator. This is a nonlocal operator based on an integral with a singular kernel. The same conclusion can be made for the fractional integrator operator; fractional differentiation operator definition being based on the fractional integrator operator definition. This situation explains why these operators are still not well defined and that several definitions still coexist, which impedes the process of becoming standard tools. Since the first recorded reference work in 1695 up to the present day, many articles have been published on this subject, but much progress is still to be done particularly on the relationship of these different definitions with the physical reality of a system (through taking into account the initial conditions for instance).

A fractional order system is a system described by an integro-differential equation involving fractional order derivatives of its input(s) and/or output(s). From a physical point of view, linear fractional order systems are not quite conventional linear systems, and not quite conventional distributed parameter systems. They are in fact halfway between these two classes of systems. Fractional order systems exhibit long memory or hereditary effects. Hence, they are a modelling tool well suited to a wide class of phenomena with nonstandard dynamic behaviour and the applications of fractional order systems are now well accepted in the following disciplines:

(i) electrical engineering (modelling of motors, modelling of transformers, skin effect, etc.);

(ii) electronics, telecommunications (phase-locking loops, etc.);

(iii) electromagnetism (modelling of complex dielectric materials, etc.);

(iv) electrochemistry (modelling of batteries and ultracapacitors, etc.);

(v) thermal engineering (modelling and identification of thermal systems, etc.);

(vi) mechanics, mechatronics (vibration insulation, etc.);

(vii) rheology (behaviour identification of materials, etc.);

(viii) automatic control (robust control, system identification, observation and control of fractional systems, etc.);

(ix) robotics (modelling, path tracking, path planning, etc.);

(x) signal processing (filtering, restoration, reconstruction, analysis of fractal noises, etc.);

(xi) image processing (fractal environment modelling, pattern recognition, edge detection, etc.); 
(xii) biology, biophysics (electric conductance of biological systems, fractional models of neurons, muscle modelling, etc.);

(xiii) physics (analysis and modelling of diffusion phenomenon, etc.);

(xiv) economy (analysis of stock exchange signals, etc.).

The goal of the present special issue is to give an overview of recent results obtained in the field. This special issue is only a sample of the work carried out throughout the world, but we hope it is representative of the broached themes and is a useful source of information to begin with fractional differentiation and its applications or to develop new researches. The 19 papers are grouped in six major areas:

(i) mathematical tools, analytical and numerical solutions, and approximation of fractional order systems,

(ii) fractional order systems properties analysis,

(iii) applications in system modelling,

(iv) applications in system identification,

(v) applications in control theory and robotics,

(vi) applications in signal and image processing.

Jocelyn Sabatier Clara Ionescu József K. Tar

José A. Tenreiro Machado 


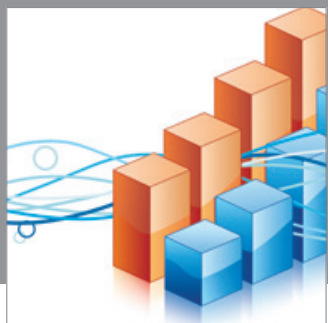

Advances in

Operations Research

mansans

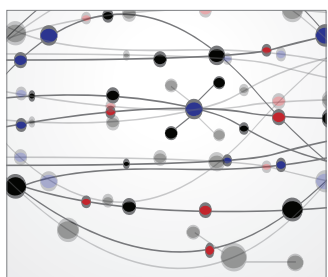

The Scientific World Journal
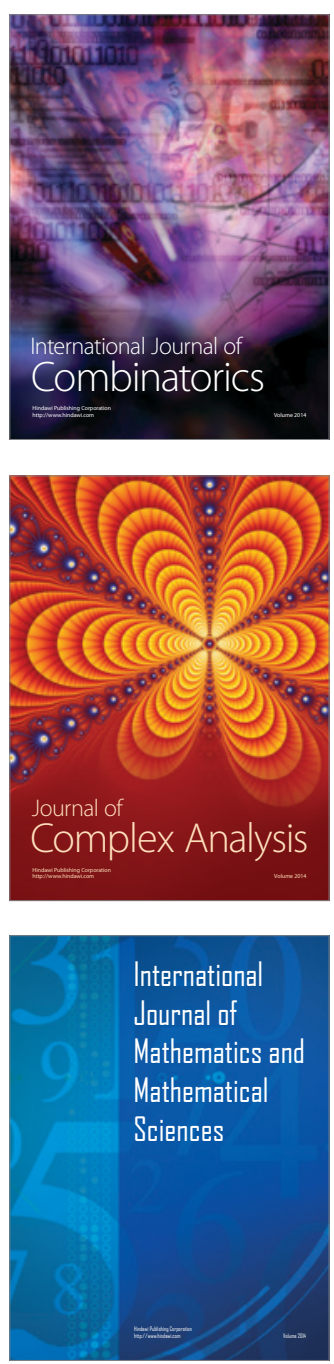
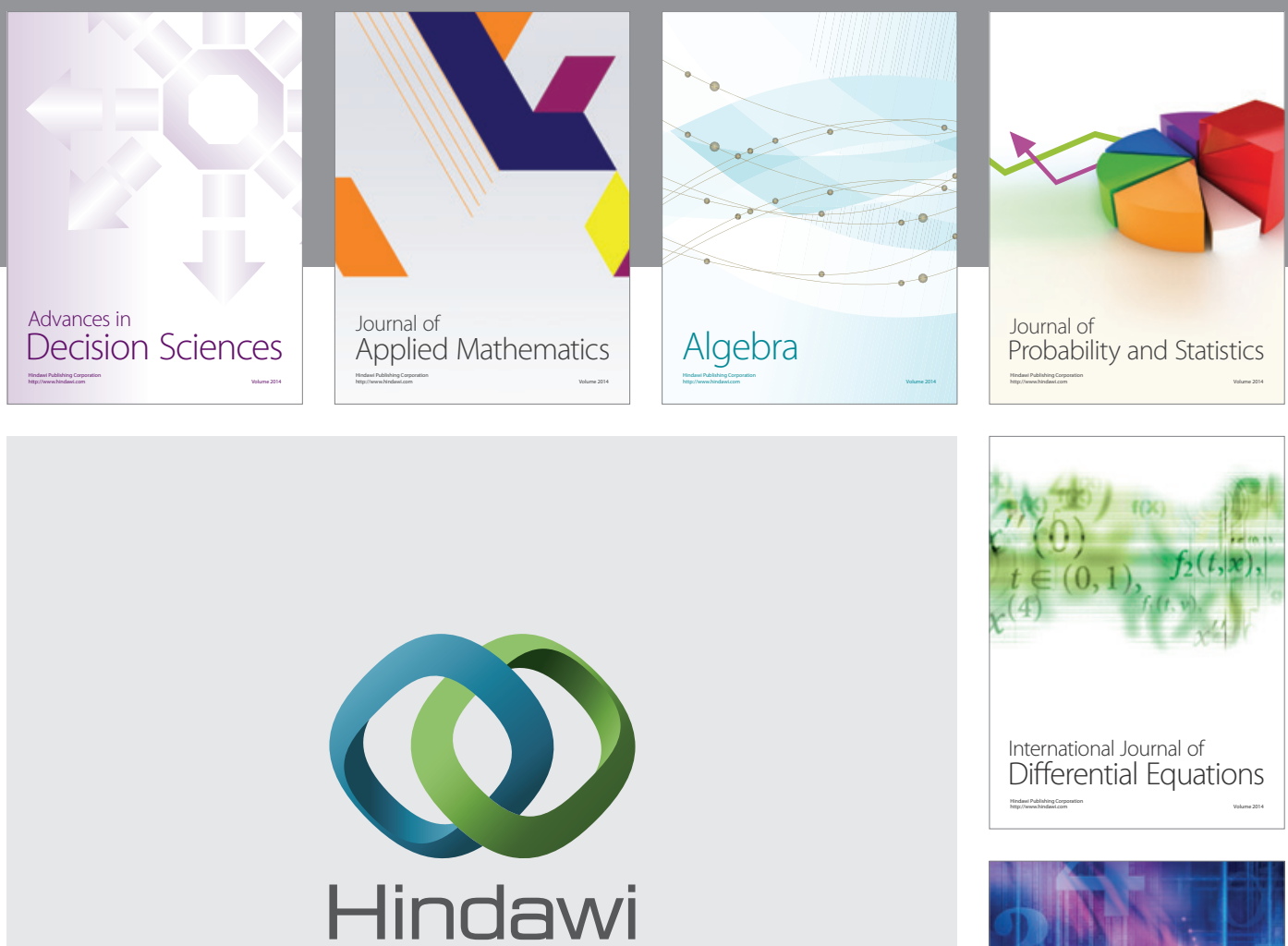

Submit your manuscripts at http://www.hindawi.com
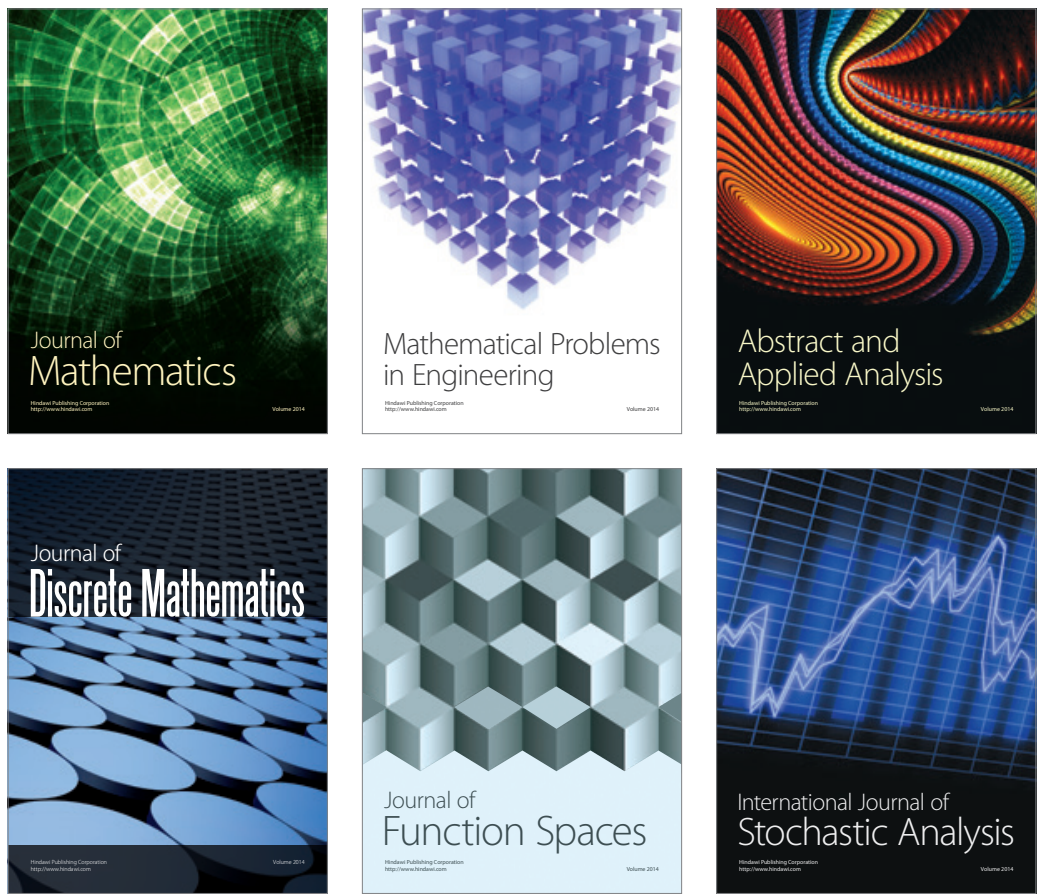

Journal of

Function Spaces

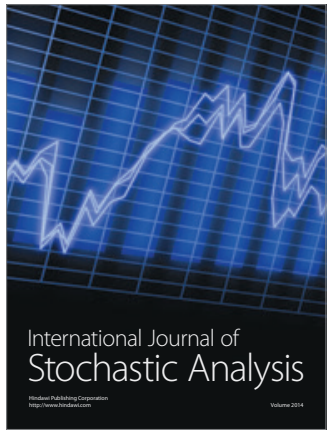

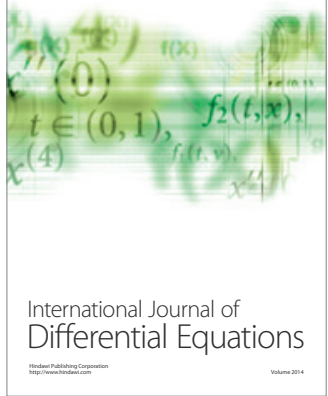
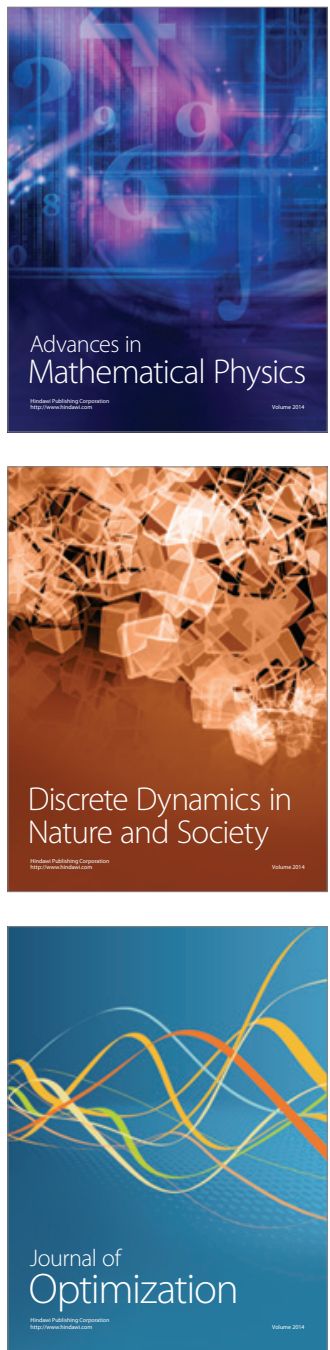